\title{
Contextos socioeducativos de prácticas y sus aportes a la formación pedagógica del futuro profesorado ${ }^{1}$
}

\section{Socio-educational contexts of teaching practices and their contributions to pedagogical training of future teachers}

\section{Contextos socioeducativos de práticas e suas contribuições para a educação pedagógica dos futuros professores}

\author{
Carolina Flores-Lueg* \\ Omar Turra-Díaz**
}

\begin{abstract}
RESUMEN
La formación práctica instalada en los planes de estudio de la Formación Inicial Docente (FID) se concibe como un espacio curricular fundamental para el aprendizaje de la enseñanza y la construcción de conocimientos pedagógicos en el futuro profesorado. Considerando que el desempeño profesional docente se desarrolla en contextos demográficos y socioculturales diversos, se presentan en este artículo resultados de una investigación que indagó en las significaciones que atribuye el estudiantado de pedagogía a la experiencia de formación práctica en dos contextos socioeducativos distintos: la escuela
\end{abstract}

1 Este artículo presenta resultados del proyecto Grupo de Investigación PROFOP, Profesorado: Políticas de formación y praxis profesional (Código: GI171123/EF, 2017-2019) y del proyecto regular "La práctica profesional como escenario para la construcción de saberes pedagógicos desde las voces de estudiantes, profesores guías y supervisores" (Código 182823 2/R), ambos financiados por la Dirección de Investigación de la Universidad del Bío-Bío.

*Universidad del Bío-Bío. Chillán, Chile. E-mail: cflores@ubiobio.cl. https://orcid.org/00000001-5219-0617

**Facultad de Educación y Humanidades. Programa Doctorado en Educación Universidad del Bío-Bío, Universidad Católica de Temuco, Chile. E-mail: oturra@ubiobio.cl. https://orcid. org/0000-0001-5000-2336 
urbana y la escuela rural. En su diseño, se utilizó una metodología cualitativa, siendo la entrevista semiestructurada la técnica de producción de información. Participaron doce estudiantes pertenecientes a dos carreras de pedagogía de naturaleza generalista, de una universidad pública de Chile. Entre los resultados principales se destaca una positiva valoración de la formación práctica en ambos contextos de desempeño, y en particular, para el caso de la ruralidad, la inmersión formativa en este espacio educativo contribuye a ampliar los significados acerca del ser docente, relevando el rol social y el trabajo con la comunidad que desarrollan el profesorado en este contexto.

Palabras claves: Formación de profesores. Formación práctica. Contextos socioeducativos. Escuela urbana. Escuela rural.

\begin{abstract}
Practical training installed on the curriculum of initial teacher instruction is understood as a fundamental curricular space for teaching learning and the construction of pedagogical knowledge in future teachers. Considering that the professional teacher performance is developed in diverse demographic and sociocultural contexts, this article presents the results of a research that looked into the significances attributed by pedagogy students to the experience of practical training in two different socio-educational contexts: the urban and rural school. In its design, it was employed a qualitative methodology, the semi-structured interview was the technique of production of information. Twelve students of two General Primary Education programs, from a public university in Chile participated. Among main results, a positive valuation of practical training is highlighted in both performance contexts, and in particular, for rurality, the formative immersion in this educational space contributes to extend the meanings about being a teacher, relieving social role and work with the community that teachers develop in this context.

Keywords: Teachers training. Practical training. Socio-educational contexts. Urban school. Rural school.
\end{abstract}

\title{
RESUMO
}

A formação prática instalada nos currículos da Formação Inicial de Professores (FID), é concebida como um espaço curricular fundamental para a aprendizagem do ensino e a construção do conhecimento pedagógico nos futuros professores. Considerando que o desempenho profissional docente tem lugar em vários contextos demográficos e socioculturais, são apresentados neste artigo resultados de pesquisa que explorou os significados atribuídos pelos alunos de pedagogia para experimentar a formação prática em dois 
contextos socioeducativos diferentes: a escola urbana e a escola rural. Em seu delineamento, utilizou-se uma metodologia qualitativa, sendo a entrevista semiestruturada a técnica de produção de informação. Doze estudantes pertencentes a duas carreiras pedagógicas de caráter generalista de uma universidade pública do Chile participaram. Entre os principais resultados de uma avaliação positiva da formação prática em ambos os contextos de desempenho se destaca, em particular, para o caso de ruralidade, imersão formativa neste espaço educacional ajuda a expandir os significados sobre ser um professor, assumir o papel social e trabalhar com a comunidade que os professores desenvolvem neste contexto.

Palavras-chave: Formação de professores. Treinamento prático. Contextos socioeducativos. Escola urbana. Escola rural.

\section{Introducción}

Las políticas de modernización de la formación inicial docente, impulsadas en Chile desde hace más de dos décadas, enfatizan la necesidad de insertar temprana y progresivamente a los futuros pedagogos en el centro escolar, como una forma de acercarlos al quehacer docente y a la realidad del aula. Esta estrategia se oficializa a partir del Programa de Fortalecimiento de la Formación Inicial Docente, promovido por el Ministerio de Educación (MINEDUC), entre los años 1997-2001. Entre las principales iniciativas que contenía el Programa, se encontraba aquella que "inducía" a las instituciones formadoras de profesores a realizar procesos de renovación curricular, cuyo énfasis se orientó, entre otros aspectos, a la definición de una línea formativa específica vinculada a la práctica desde el inicio del proceso formativo, la que adquiriría un carácter sistemático y progresivo.

Es así que la formación práctica dispuesta en los planes de estudio de la formación inicial docente se visualiza como un espacio curricular clave para el aprendizaje de la enseñanza y para la adquisición de conocimientos pedagógicos en el futuro profesorado, por cuanto las interacciones sociales y educativas que logren vivenciar en escenarios reales, se estiman como fundamentales para los procesos de construcción de aquellos saberes que servirán de base para su ejercicio profesional (MARCELO, 2001; HIRMAS y CORTÉS, 2015). Bajo esta premisa, la práctica adquiere un significado especial en los planes de estudio y en los perfiles de egreso de la Formación Inicial Docente (FID), en tanto se configura como un continuum curricular que busca vincular tempranamente el espacio académico con el espacio escolar, entre otros aspectos. La articulación 
que se logre generar entre ambos escenarios, permitiría al futuro profesorado adquirir los insumos pedagógicos propios del desempeño docente, en tanto se trasformaría en una instancia clave para aprender a enseñar y construir desde ahí un saber pedagógico, como consecuencia de la puesta en común de un conocimiento proposicional (saber teórico-académico) o formalizado, y un conocimiento práctico (saber en y sobre la acción docente) de carácter procedimental (VEZUB, 2016).

No obstante, la investigación especializada en el ámbito de la formación práctica (HIRMAS y CORTÉS, 2015; LABRA, 2011; MONTECINOS et al, 2011), evidencia una serie de tensiones que persisten entre la concreción del perfil de profesor, que se promueve desde las políticas educativas, y los requerimientos que demanda el sistema escolar, donde las instituciones de educación superior que ofrecen carreras de pedagogía tendrían una importante responsabilidad al respecto.

Entre los cuestionamientos levantados hacia la FID, y concretamente sobre los conocimientos prácticos que se logran obtener en esa etapa formativa, se observan algunas falencias dignas de destacarse: una comprensión inadecuada de la naturaleza teórico-práctica, propia de la acción docente (HIRMAS y CORTÉS, 2015), donde la práctica es simplemente es concebida como cualquier actividad formativa que relaciona al estudiantado de pedagogía con un centro educativo, observándose en la realidad que "hay una amplia variabilidad en la forma de concebir el concepto de práctica y el propósito del contacto con el centro educativo" (CONTRERAS et al., 2010, p. 92); el rol distante que se percibe desde las instituciones universitarias hacia los centros escolares, respecto al escenario de las prácticas (LATORRE, 2009; MONTECINOS y WALKER, 2010), lo que también se expresa en una distancia conceptual entre un "saber experto" de la academia y un "saber práctico" del profesorado (TURRA-DIAZ y FLORES-LUEG, 2018).

Otra debilidad que se ha incubado en la formación práctica de futuros pedagogos, según nuestras investigaciones (TURRA-DÍAZ, FERRADA y VILLENA, 2013; TURRA-DÍAZ y FLORES-LUEG, 2018), es la ausencia de inserción de practicantes en la diversidad de escenarios socioeducactivos en los que podrían desarrollar su labor docente, pues las instituciones formadoras fundamentalmente intencionan las prácticas pedagógicas en centros educativos localizados en realidades urbanas. De esta manera, se pueden caracterizar como excepcionales las carreras de pedagogía del país que consideran el escenario rural o indígena como una instancia y espacio de prácticas, que ofrecen oportunidades para la construcción y reconstrucción de conocimientos pedagógicos en los futuros profesores

Ahora bien, si se tiene en consideración la relevancia que adoptan las vivencias que pueden obtener los futuros pedagogos en los contextos escola- 
res, valorados como escenarios claves para la construcción de conocimiento profesional (HIRMAS y CORTÉS, 2015), a nuestro juicio, resulta de interés investigativo indagar en las percepciones sobre las experiencias de práctica que portan estudiantes de pedagogía, particularmente aquellos que han tenido la oportunidad de estar inmersos en centros escolares caracterizados por su diversidad socioeducativa. Es por ello que, en este artículo presentamos los principales resultados de una investigación que tuvo como propósito develar las significaciones pedagógicas atribuidas a la experiencia formativa de práctica, en dos contextos escolares claramente distinguibles - urbanidad, ruralidad-, desde el estudiantado de dos carreras de pedagogía de corte generalista.

\section{Marco Teórico}

\section{El área práctica en la Formación Inicial Docente}

Se ha planteado que el desarrollo profesional docente es el resultado de un cúmulo de experiencias y conocimientos vinculados con dimensiones formativas y al ejercicio de la profesión. Para Day (2005), este conocimiento y desarrollo profesional se produce en tres entornos específicos: en el aprendizaje directo alcanzado con la asistencia a cursos, seminarios, talleres, etc., en el aprendizaje en la escuela por el trabajo y colaboración desarrollada con otros docentes y en el aprendizaje logrado fuera de la escuela, colaborando con grupos de otros niveles e instancias educativas.

No obstante, también existe consenso en que la etapa formativa inicial resulta fundamental en el proceso de profesionalización docente, puesto que aquí se asientan las bases de un saber hacer pedagógico que provee de herramientas para el futuro desempeño profesional. Por ello, la línea de formación práctica se representa, en los actuales programas formativos del profesorado, como una instancia clave y propicia para el aprendizaje de la profesión docente (GONZÁLEZ y FUENTES, 2011). Sin embargo, su implementación en la formación universitaria responde a una variedad de comprensiones y/o entendimientos de la relación teoría-práctica, presentándose de este modo una ausencia de un modelo unificado (ZABALZA, 2011).

La experiencia práctica, para el caso de los estudiantes de pedagogía, configura un saber que también se ha denominado "saber docente", el que en su conformación comprende aspectos formalizados y otros de carácter procedimental (VEZUB, 2016), resultante de la interacción con el entorno, de la reflexión 
que realizan sobre su desempeño y vinculación con los aspectos conceptuales adquiridos en la formación inicial. Es en esta instancia curricular donde se despliega y relaciona el conocimiento teórico con el práctico y en donde se produciría un saber pedagógico a la base de procesos reflexivos.

En consecuencia, la práctica, como actividad curricular, se concibe como un espacio privilegiado para que estudiantes de pedagogía adquieran el conocimiento acerca de la enseñanza a partir de los saberes construidos por un profesor guía, vale decir, de sus experiencias, reflexiones y representaciones (TARDIF, 2010). Esta emergencia y reconocimiento del saber generado en el ámbito práctico, viene también a revalorizar la idea de que este conocimiento pedagógico es situado y contextual, que resulta imprescindible para el ejercicio docente.

\section{La urbanidad/ruralidad como escenario de la formación práctica}

Los contextos socioculturales en que se desarrolla el proceso de enseñanza-aprendizaje, contienen características específicas y diferenciadas que hacen del hecho educativo un proceso también diferenciado. En este sentido, el conocimiento pedagógico y disciplinar que se promueve desde las instituciones formadoras entra en contacto, durante la formación práctica, con unos saberes y unas prácticas educativas que emergen de los contextos sociales, culturales y territoriales de la que los docentes experimentados ya han incorporado como un conocimiento profesional, en consideración a sus años de ejercicio profesional en estos escenarios socioeducativos.

Un primer aprendizaje al respecto, es desarrollar la capacidad de entender que la enseñanza es un proceso complejo y diverso, vinculado a un contexto sociocultural específico, en la que participan sujetos con características, pretensiones y expectativas distintas. Es así como los procesos educativos en una realidad urbano-marginal está cruzada por complejidades y factores propios derivados de la vulnerabilización socioeconómica (FERRADA, VILLENA y TURRA, 2015) y la enseñanza en la ruralidad comprende otros factores tan complejos y múltiples como los que se encuentran en la realidad urbana.

Como lo ha planteado la investigación educativa, la práctica en un contexto social y cultural claramente diferenciado como lo es el escenario educativo rural, permite a los pedagogos en formación, acercarse a la labor docente desde una perspectiva diversa, con significaciones y valoraciones elaboradas desde la muldimensionalidad, propias y/o características del desempeño en ruralidad donde la práctica pedagógica trasciende al ámbito del espacio escolar para configurarse en un campo de acción vinculado a componentes institucionales, sociales y culturales (LORENZATTI, BRUMAT y BEINOTTI, 2014). También se sabe que una de las características singulares que presentan mayoritariamente 
las escuelas ubicadas en contextos rurales corresponde al llamado multigrado, vale decir un espacio escolar en el que se insertan y conviven niños y niñas de edades diferentes, con grados de desarrollo y de conocimiento distintos, en cursos que integran niveles educativos disímiles, todos ellos a cargo de un solo profesor (BOIX y BUSTOS, 2014). Esta condición implica que el docente debe ser capaz de llevar a cabo una práctica pedagógica diferenciada, buscando dar respuesta a la heterogeneidad del aula. No obstante, esta situación puede generar cierto nivel de complejidad o desequilibrio para la labor docente en cuanto a la implementación de metodologías de enseñanza diferenciadas, específicamente en lo referido al planteamiento didáctico y a la organización de aula. Sin embargo, la diversidad de niños y niñas también puede ser observada como una oportunidad de intercambio de conocimientos e información entre ellos mismos, lo que promueve la socialización y el aprendizaje (BOIX y BUSTOS, 2014), por cuanto los más grandes actúan como pares mediadores al interactuar con los más pequeños, donde todos pueden aprender de todos, independiente del nivel educativo en el que se encuentren. En este sentido, las características del aula multigrado pueden constituirse en un espacio que favorece la experimentación educativa y el desarrollo de una pedagogía activa y contextualizada, al mismo tiempo que permite contar con un sistema de enseñanza concéntrico y flexible (VERA, OSSES y SCHIEFELBEIN, 2012).

\section{Marco Metodológico}

El estudio se realizó en base a los principios investigativos del paradigma comprensivo-interpretativo, específicamente desde una metodología cualitativa, en tanto propone alcanzar comprensión sobre una realidad investigada desde las comprensiones-significaciones de los propios sujetos que participan del campo objeto de investigación (FLICK, 2012). En el marco de esta metodología, se utilizaron algunos de los postulados del método conocido como Teoría Fundamentada (GLASER y STRAUSS, 1967).

La investigación consideró estudiantes de dos carreras de pedagogía de la Universidad del Bío-Bío, ubicada geográficamente en la ciudad de Chillán, Región de Ñuble-Chile. Esta Universidad es de carácter estatal (entre 18 de este tipo que existen en el país) y forma parte del Consejo de Rectores de Universidades Chilenas (CRUCH), que agrupa a 25 instituciones de Educación Superior con mayor tradición y prestigio en el país. Actualmente, se encuentra acreditada por un periodo de 5 años (de un máximo de 7) por la Comisión Nacional de Acreditación (CNA). 
Los participantes de la investigación fueron seleccionados a partir de los siguientes criterios de inclusión: estudiantes de las carreras de pedagogía de carácter generalista, puesto que desarrollan prácticas pedagógicas desde el II semestre de sus estudios; que cursen el último año de su formación y que acepten participar voluntariamente del estudio. En base a estos criterios se conformó un grupo de doce estudiantes, 6 de Pedagogía en Educación Parvularia (EP) y 6 de Pedagogía en Educación General Básica (PEGB). La totalidad de las participantes son mujeres y todas se encontraban cursando el VIII semestre del plan de estudio, correspondiente a la última etapa formativa.

La técnica de producción de información utilizada fue la entrevista semiestructurada, pues permite la realización de preguntas emergentes para aclarar o profundizar las comprensiones de los participantes, siguiendo un guion de preguntas preestablecidas. La entrevista se realizó a cada participante en forma directa, a quienes se informó sobre la finalidad del estudio y se les aseguró la confidencialidad de los datos. El discurso fue grabado en formato de audio y posteriormente transcrito en forma literal. Para el análisis de la información se empleó el software Atlas.ti, versión 7.0, generándose una Unidad Hermenéutica en base al contenido de los documentos transcritos. Se realizó un proceso de codificación mixta, posteriormente se determinó la coocurrencia de códigos y en base a estos resultados se generaron dos familias de códigos (ver Figura 1), las que se constituyeron en las categorías centrales de análisis.

\section{FIGURA 1 - CATEGORÍAS Y RED CÓDIGOS}

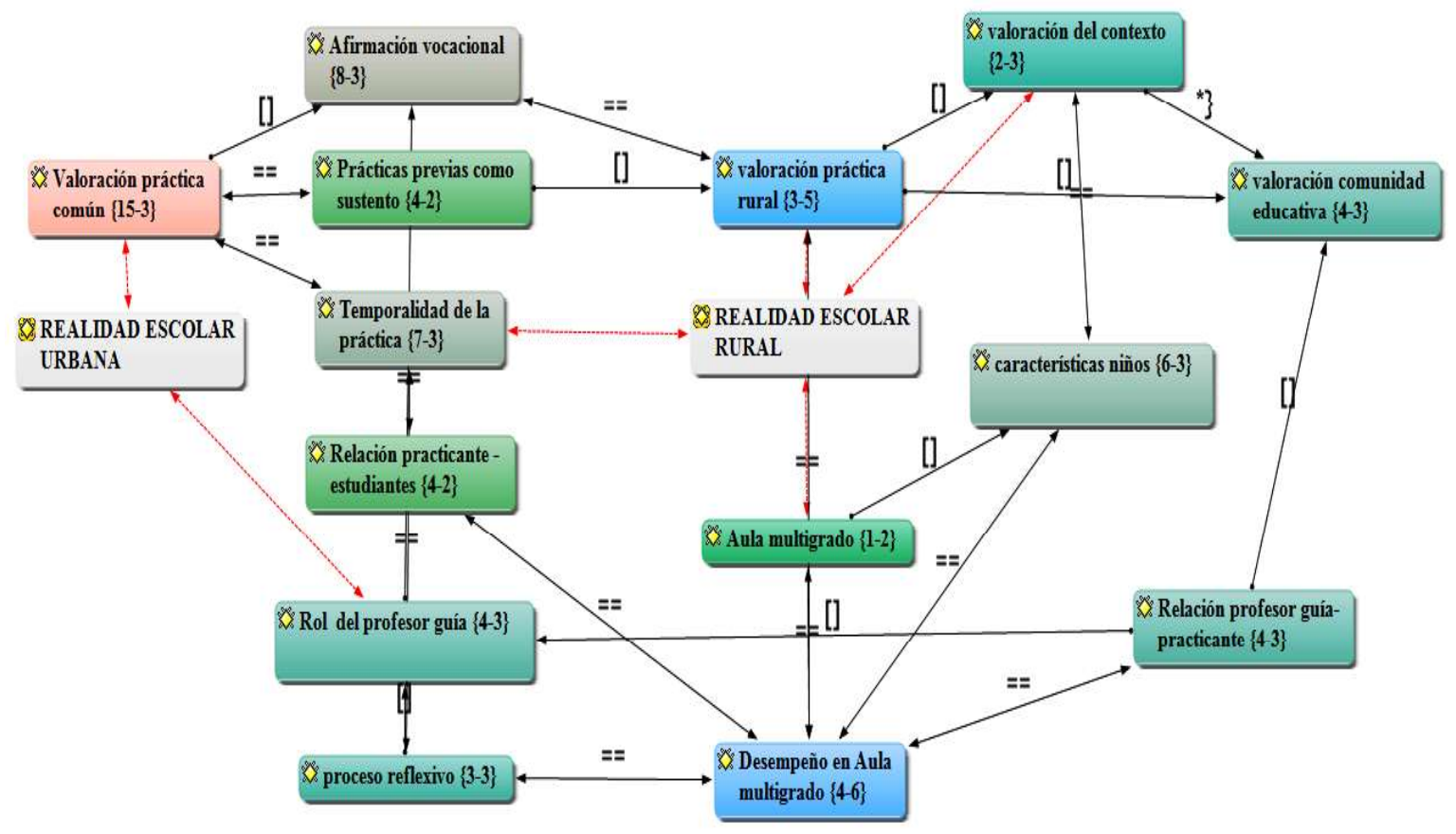

FUENTE: Elaboración propia con Atlas.ti v. 7 


\section{Resultados}

Los resultados se exponen en consideración a los contextos escolares en los cuales las estudiantes realizan sus prácticas pedagógicas y la práctica profesional. El primero de ellos y el más cercano es la realidad escolar urbana, como escenario de práctica común para ambas carreras consideradas en el estudio, el que además responde al contexto habitualmente definido por la universidad para la realización de esta trascendental actividad curricular. El otro espacio corresponde al contexto rural donde, particularmente, estudiantes de la carrera de Pedagogía en Educación General Básica desarrollan una práctica en modalidad de internado durante un mes.

\section{Realidad escolar urbana, como escenario de práctica común}

La práctica llevada a cabo en centros escolares urbanos es altamente valorada por las pedagogas en formación, pues el hecho de insertarse en un aula real aporta de manera significativa a su proceso formativo en diversos aspectos, entre ellos, les permite afirmar su vocación profesional y acercarse a la diversidad de situaciones de enseñanza y aprendizaje que emergen de la realidad áulica. Asimismo, la inmersión en el escenario escolar en forma temprana y sistemática se transforma en una instancia que no solo les ofrece la posibilidad de construir conocimientos pedagógicos de naturaleza práctica, sino que al mismo tiempo las situaciones de aula vivenciadas, las lleva también a cuestionar la pertinencia en la práctica de los conocimientos teóricos adquiridos en la formación.

Como oportunidad formativa, la práctica contribuye a la afirmación profesional y a visualizar las complejidades del aula. Al respecto, en perspectiva de las estudiantes, esta actividad es altamente valorada porque permite una aproximación a su futuro rol docente y, como consecuencia de ello, aporta a la definición de su vocación profesional. A partir del acercamiento que comienzan a tener con el ambiente escolar y la dinámica que en ese espacio se percibe, logran poner a prueba sus representaciones previas sobre el quehacer docente y valorar sus aptitudes personales para la labor pedagógica, lo que resulta ser concluyente en términos de encantarse con la profesión, o bien, sufrir una desilusión que les lleve a abandonar la carrera en sus inicios, 
Es relevante, puesto que nos acercamos desde un comienzo a la realidad del ejercicio docente y podemos ver in situ cómo se da el proceso enseñanza - aprendizaje (PEGB: 5).

Agradezco que tenga práctica desde primer año, porque nos sirve para darnos cuenta si realmente nos gustaría seguir con la carrera o no, es que para mí fue la primera etapa de práctica darme cuenta de si era lo mío o no (EP: 4).

Importa en lo que respecta al encantamiento, en que te guste la carrera. Yo creo que es el primer foco, para ser además resiliente y decir quizás no fue tan maravilloso lo que me plantearon, pero si yo no tengo esa capacidad o ese encantamiento también puedo desertar, es tan drástico como eso y por eso hay muchas personas que desertan el primer año de carrera o en lo sucesivo (EP: 3 )

Yo creo que la práctica desde primero permite el encantamiento, porque en tercero ya es tarde para una darse cuenta de que realmente no está encantado con la carrera (EP: 1).

También, la instancia de práctica se constituye en una experiencia que contribuye a la generación de procesos reflexivos sobre su propio desempeño y sobre el quehacer docente en general. No obstante, para las pedagogas en formación, la potencialidad de estos procesos se encuentra en estrecha vinculación con condiciones de carácter personal y a la temporalidad de esta actividad, destacándose en este último aspecto que el tiempo de permanencia en el centro escolar y la frecuencia de asistencia al aula favorecería la reflexión, pero ésta lograría alcanzar mayor profundidad en la práctica profesional, debido a que es la instancia que les permite un mayor espacio de actuación,

Soy bien reflexiva en general en mi vida, entonces yo reflexiono mucho sobre las cosas y eso también es de cada uno, es particular (EP: 5).

En práctica profesional más aún, porque una ya conoce a los niños y conoce al equipo, entonces una cuestiona sobre lo que hace, reflexiona en forma más profunda, más elaborada y más crítica, porque una quiere ir mejorando cada vez más (EP: 1).

Como es la práctica profesional, uno está todos los días, entonces a lo mejor uno antes podía hacer esa reflexión, pero uno antes no podía hacer un gran cambio si se iba una vez a la semana o tres veces, pero ahora uno está todos los días con ellos, entonces una ya puede ir buscando estrategias y haciendo cosas como para poder innovar (EP: 3). 
Por otra parte, el itinerario de prácticas que han tenido las estudiantes a lo largo de su proceso de formación es visualizado como una instancia propicia para ir construyendo paulatinamente sus propios conocimientos pedagógicos, a la base de la secuencialidad de la inserción en las aulas escolares, argumentando que lo que se ha aprendido en una práctica anterior les ha servido de base para enfrentar con mayor seguridad una nueva actividad de práctica,

El realizar prácticas todos los semestres a lo largo de la carrera, sirve de forma personal para aprender lo bueno y malo al realizar las clases, cosa que traté de aplicar ahora (PEGB: 1).

Hay muchas cosas que yo he aprendido que no se dan aqui en la universidad. Me refiero al aspecto propio, yo como veo mi avance desde el primer año, porque nuestra carrera tiene práctica desde el segundo semestre del primer año. Ahora que ya estoy en cuarto, sé que he ido en una progresión, en una evolución en mi manera de relacionarme con los niños, mi tono de voz, las palabras que utilizo, la corporalidad, los movimientos (PEGB: 6).

Claramente las prácticas realizadas antes me sirvieron para hacer las clases en la ruralidad, el realizar prácticas todos los semestres a lo largo de la carrera, sirve de forma personal para aprender lo bueno y malo al realizar las clases (PEGB: 1).

Es importante destacar que la inmersión en el aula escolar, también, se ha transformado en una instancia que les ha llevado a cuestionar la formación académica recibida en la universidad, en tanto manifiestan que en el desarrollo de sus prácticas se han visto enfrentadas a la necesidad de dar respuesta a fenómenos para los que no ha recibido formación, o bien, poseen una insuficiente preparación para su abordaje. Entre ellos, destacan la atención de niños o niñas con necesidades educativas especiales y ciertas dificultades referidas a la capacidad para liderar el proceso pedagógico, fundamentalmente en lo que respecta a la resolución de conflictos.

Del mismo modo, manifiestan que la aplicación de la teoría en situaciones prácticas no siempre es posible, aludiendo a que ésta representa un ideal y que muchas veces no les permite dar respuesta a las complejidades presentes en el aula. Esta tensión, las lleva a validar el aporte que realiza el profesor guía para el proceso de formación, 
yo creo que a lo largo de la formación debería tratarse ese tema, el tema de las relaciones humanas, porque a veces pasa que se dan las situaciones emergentes y no tienes idea cómo actuar frente a conflictos (EP:1). Cuando me toca realmente estar en una situación, por ejemplo, niños con trastornos especificos del lenguaje, acá en la universidad se da una pincelada de lo que significa entre comillas este problema, pero cuando uno lo tiene en la práctica en la realidad tiene que necesariamente buscar más información porque hay muchas cosas que uno desconoce (EP:4). Aqui en la universidad nosotros vemos lo que es ideal de práctica, entonces vemos teóricamente un mundo maravilloso, pero cuando llegamos a la realidad como que no es todo tan maravilloso y ahí nos damos cuenta de que no toda la teoría se lleva a la práctica como debería ser (EP: 6). La práctica permite cuestionar la teoría, aunque también te sirve, no es que no sirva nada, igual es fundamental porque nos da un marco, una referencia. Obviamente por la teoría no vamos a forzar ciertos aspectos, solo porque lo dice la teoría (EP: 2).

En muchos casos, son más significativos los profesores de las escuelas más que los de la Universidad (PEGB: 4).

A pesar de las anteriores consideraciones, desde las voces de las estudiantes se desprende con claridad que las debilidades observadas son fruto de su inmersión en el aula escolar, en la complejidad de su realidad cotidiana, por lo que manifiestan una positiva valoración de la práctica como área de formación,

Un profesional no sería profesional si solo ve teoría y no va donde pasan las cosas, si no ve la realidad, estarías construyendo algo en el aire porque uno donde realmente aprende es en la práctica (EP: 4).

\section{Realidad escolar rural y su aporte a la formación inicial docente}

La inmersión en la ruralidad se transforma en una instancia de desempeño que adopta una valoración especial para las participantes del estudio. En consideración a una nueva experiencia formativa como es la de interactuar con otros actores de esta realidad educativa, esto es, las familias y miembros de la comunidad circundante, fue lo que permitió construir nuevos significados respecto del ser docente y de la responsabilidad social de la escuela. Se agrega a lo 
anterior, la apertura a una nueva experiencia de actuación pedagógica, como es el desempeño en un aula multigrado, particularmente extendida en los centros educativos localizados en ruralidad.

A una comprensión inicial del ser docente vinculada, fundamentalmente, con el proceso de enseñanza y aprendizaje, la inmersión en la escuela rural contribuye a ampliar los significados acerca de lo que significa ser profesor/a, relevando el rol social y el trabajo con la comunidad que desarrollan los docentes en este contexto,

Sirvió para ver desde otra perspectiva la educación y como le toman mayor importancia a ésta, tanto los estudiantes como los apoderados, siendo realmente una experiencia valorable, gratificante y agradable debido a la cercanía y compromiso de la unidad educativa (PEGB: 1). La práctica rural es un espacio para desenvolverse en un contexto que, como su nombre lo dice, se encuentra en un campo, donde el entorno, el proceso de enseñanza, los alumnos y los apoderados son algo muy distinto a lo que acostumbramos a vivir estando en una escuela de ciudad (PEGB:2).

Las docentes en formación resaltan las específicas características que presenta el contexto rural, tanto desde su dimensión física/geográfica como sociocultural. Desde este último aspecto, reconocen la acogida y el apoyo que logran obtener de las familias en la promoción de aprendizajes en los niños y en la labor pedagógica en general. Del mismo modo, resaltan positivamente las particularidades que presentan los estudiantes que viven en ruralidad, aludiendo a cualidades como la inocencia, la motivación por ir a la escuela y aprender, el respeto hacia el otro y, principalmente, hacia la figura del profesor/a, entre otras. Características que, al decir de ellas, no siempre están presentes en los niños y niñas que asisten a establecimientos urbanos,

Trabajar en un ambiente rodeado de naturaleza, arboles, ríos, animales es totalmente gratificante. Se dispone de todo un terreno donde los niños pueden jugar libremente en sus recreos, correr grandes distancias, gritar sin que los callen, pasarlo bien (PEGB: 2).

La cercanía y compromiso de la unidad educativa, tomando en cuenta la personalidad de los niños, viviendo una niñez diferente a la realidad que he visto en la ciudad, eran inocentes, empáticos, no existían las burlas, entre otros valores que inculcaba la escuela (PEGB: 1). 
Aquí los niños son realmente niños; no están expuestos al mundo urbano, la discriminación no existe, juegan entre todos, se ayudan entre sí, respetan y valoran a los más grandes, a sus profesores. (PEGB: 2).

Los apoderados también juegan un rol importante en esta valoración; son muy comprometidos con el aprendizaje de sus hijos [...], todos se conocen y son totalmente comprometidos en trabajar para la escuela” (PEGB:4).

Las futuras pedagogas también exponen una positiva valoración sobre las implicancias que tiene para su proceso formativo insertarse en un aula multigrado, destacando, por ejemplo, que la reducida cantidad de estudiantes en la sala de clases les permite llevar a cabo una práctica pedagógica más personalizada y pertinente a los requerimientos individuales. No obstante, la diversidad de niveles de desarrollo que interactúan en forma simultánea en el aula y las necesidades particulares, también se constituye en una condición que les genera tensión, asociada a ciertas dificultades técnicas, entre ellas, la obligatoriedad de tener que planificar en forma diferenciada y, además, poner a prueba estrategias diversificadas de enseñanza para dar respuesta a las necesidades de cada subgrupo de niños,

Hacer clases en un curso multigrado también fue algo relevante de esta práctica, puesto que al momento de planificar debía ser contextualizado, $y$ además hacer lo mejor posible para que los estudiantes de distintas edades y niveles se integraran de una manera correcta a la clase, y que fuera armónica para todos (PEGB:1).

Es la complejidad en las que nos vemos como docentes en formación, teniendo seis cursos en una sala y tratar de enlazar los contenidos y objetivos para hacer algo más integral (PEGB: 5).

Nos permitió tener una mayor cercanía con los niños y sus requerimientos como personas individuales, cada uno demandaba una estrategia de enseñanza y aprendizaje distinta, al ser cada uno de un nivel distinto dentro de un mismo espacio, por lo que pudimos desenvolvernos, a la vez, de forma multifacética con cada uno de ellos de forma particular (PEGB:6).

Por otra parte, se refieren positivamente hacia el clima laboral que se genera al interior del centro escolar y, particularmente, a las dinámicas que presentan las relaciones interpersonales que logran establecer entre los miembros de la comunidad educativa, argumentando que al tratarse de una escuela unidocente, donde la labor directiva y pedagógica la desempeña simultáneamente el mismo 
profesor junto a un reducido personal de apoyo, se favorece la generación de un ambiente armónico y acogedor. Esta situación ayuda a romper con la rigidez de los tiempos destinados al proceso de enseñanza y aprendizaje, porque, de acuerdo a lo que manifiestan las estudiantes, permite manejar mayores márgenes de flexibilidad en las distintas actividades.

También es importante destacar que el formato de "internado", propio de esta práctica en ruralidad, y el tiempo destinado para su desarrollo, otorga a las futuras docentes la posibilidad de valorar positivamente la relación que logran entablar con el profesor guía y con los mismos niños y niñas del aula, destacando sentir mayor cercanía con ellos, producto de la inmersión completa dentro de la realidad de la escuela y de la comunidad. Aquello, les permite vivenciar in situ las situaciones cotidianas que tienen lugar en dicha realidad, a diferencia de lo que visualizan en las prácticas llevadas a cabo en centros escolares urbanos, en las que solo asisten uno o dos días al aula en la semana,

...estar todo el día en el contexto rural, personalmente, sirvió para ver desde otra perspectiva la educación y como le toman mayor importancia a ésta, tanto los estudiantes como los apoderados siendo realmente una experiencia valorable, gratificante y agradable debido a la cercanía y compromiso de la unidad educativa (PEGB:1).

La relación con el profesor fue más cercana que en las prácticas anteriores ya que compartíamos todos los días durante la jornada completa de clases (PEGB:1).

Finalmente, se destaca que este escenario de práctica también contribuye al desarrollo de procesos reflexivos en y sobre la práctica. Aunque éstos no sean concebidos como tal por las estudiantes ni estén intencionados desde la realidad académica, pues el hecho de tener que tomar decisiones pedagógicas en la acción y sobre la acción, asociadas a diseñar, probar y/o validar ciertas estrategias de enseñanza, conlleva a evidenciar una práctica deliberativa sobre la factibilidad y asertividad de las decisiones tomadas, en función de dar respuesta a las necesidades individuales evidenciadas al interior del aula,

Tuvimos la oportunidad de tomar decisiones pedagógicas, de realizar nuestras propias estrategias, de aplicar el ensayo y error para ver qué era lo más factible dentro de la sala de clases (PEGB: 6). 
Por lo expuesto, la práctica en el escenario rural constituye una valiosa experiencia formativa, pues permite a los futuros docentes ampliar las significaciones respecto del ser docente y aporta a un entendimiento del desempeño profesional del profesorado en vinculación y trabajo colaborativo con las familias y la comunidad educativa, en tanto actores relevantes en el proceso educativo escolar.

\section{Discusión}

Un primer aspecto a discutir es el rol y comprensión atribuida al área de formación práctica en los planes de estudio del futuro profesorado. Al respecto, existe consenso acerca de los beneficios que conlleva el acercamiento temprano y sistemático al escenario escolar como espacio real de desempeño de la profesión docente, aspecto que, también, es relevado por los estudiantes de pedagogía. Sin embargo, desde el modelo de formación inicial docente en vigencia, aún persiste una comprensión subvalorada de esta actividad curricular, donde la formación teórica desarrollada por la academia adquiere un status superior respecto del conocimiento práctico que aportan al proceso formativo los profesores guías en el sistema escolar, como se desprende de las voces de las futuras pedagogas. Puede visualizarse una formación fragmentada entre lo teórico y lo práctico, quedando en el albedrío del estudiante el desarrollo de reflexiones articuladoras de estos campos de conocimiento. Desde este entendimiento fragmentador del proceso formativo, emerge la necesidad de remirar los modelos epistemológicos bajo los cuales se concibe la formación del profesorado en el país, para transitar a modelos que valoricen el saber docente construido desde la experiencia del desempeño, de la praxis profesional en escenarios socioculturales diversos, de tal manera que el conocimiento del profesor guía dialogue con el conocimiento teórico-académico, en la línea de avanzar hacia la co-formación del estudiantado de pedagogía. En este sentido, se puede hablar de un diálogo de saberes que se producen en dos espacios educativos distintos, pero que confluyen mancomunadamente en la formación del profesorado.

Por otra parte, el escenario de práctica permite poner a prueba los conocimientos teóricos que se promueven desde el espacio universitario, lo que favorece la generación de procesos tempranos de reflexión docente orientados a la resolución de situaciones que emergen en el aula y que se constituyen en la base de un conocimiento pedagógico situado.

Un segundo aspecto a discutir es la focalización de la formación práctica de futuros profesores en realidades escolares urbanas. Si bien la política educa- 
tiva, actualmente releva la experiencia temprana y contextual en las carreras de pedagogía, escasamente las instituciones formadoras consideran otras realidades socioculturales que no sean la escuela situada en urbanidad. Esta direccionalidad del área práctica, como actividad curricular clave en el proceso formativo, genera una aproximación parcializada a los escenarios socioeducativos que aportan conocimiento pedagógico al futuro profesorado, restringiendo, con ello, las posibilidades de acceder a un saber hacer docente propio de otras realidades de desempeño profesional, como son los contextos rurales. Es así como el aula multigrado y las especificidades culturales propias de la comunidad rural, entre otras características, transforman a la escuela en ruralidad en un escenario socioeducativo singular, cuyo potencial formativo se hace necesario valorizar y considerar en la formación inicial docente, como lo avalan los resultados de esta investigación. Por ello, consideramos relevante avanzar hacia la diversificación de los escenarios escolares de prácticas, sobre todo en aquellas regiones que presentan altos índices de ruralidad e indigeneidad, por cuanto ello permitiría disponer de un cuerpo de profesores preparados para actuar profesionalmente en contextos diversos.

Finalmente, cabe destacar la apertura en la comprensión del ser docente que emerge de la experiencia formativa de la práctica en ruralidad. La interacción con diversos actores de la comunidad circundante permitió generar, en el futuro profesorado, nuevos sentidos acerca de lo que significa la profesión docente, valorizando la dimensión social asociada al trabajo pedagógico. Esto significa que, más allá del dominio del proceso de enseñanza y aprendizaje en el aula, ciertamente relevante, la profesión docente conlleva un rol de responsabilidad social de trabajo con la comunidad en que se desarrolla la labor educativa, en tanto abarca otras dimensiones vinculadas a la identificación con el conjunto de la realidad socioeducativa, en relación a sus proyectos, dimensiones, necesidades y demandas territoriales (SORIA y HERNÁNDEZ, 2017).

\section{Conclusiones}

La formación práctica resulta ser una instancia educativa ampliamente valorada por el estudiantado de pedagogía, independiente de los contextos socioeducativos en estudio. Existe consenso que contribuye a la afirmación profesional y a visualizar las complejidades del aula, además de aportar a la generación de procesos reflexivos sobre su propio desempeño y sobre el quehacer docente en general. Particularmente, para el contexto rural se agrega un conocimiento pedagógico vinculado al trabajo en aulas multigrado, caracterizado por la he- 
terogeneidad de niveles e intereses educativos, y por un desempeño docente en vinculación y trabajo colaborativo con las familias y la comunidad circundante.

Los resultados expuestos, dan cuenta del valor atribuido al área práctica dentro del itinerario formativo del profesorado y su contribución a la adquisición de conocimientos pedagógicos-prácticos propios del desempeño docente en contexto. No obstante, se destaca por parte de las practicantes el significativo aporte que realizan los profesores del sistema escolar en su rol de guías del estudiante, rol formativo que escasamente es considerado y/o relevado desde el espacio universitario. Al respecto, postulamos que este conocimiento construido a la base de la praxis profesional constituye un valioso dato a recuperar y sistematizar por la investigación educativa, así como un insumo necesario de considerar en los programas de formación del profesorado.

\section{REFERENCIAS}

BOIX, R.; BUSTOS, A. La enseñanza en las aulas multigrado: una aproximación a las actividades escolares y los recursos didácticos desde la perspectiva del profesorado. Revista Iberoamericana de Evaluación Educativa, v.7, n.3, p.29-43. 2014.

CONTRERAS, I. et al. La escuela como espacio para aprender a enseñar: visiones desde los Programas de formación de profesores de educación media. Estudios Pedagógicos (Valdivia), v. 36, n. 1, p. 85-105, 2010. https://doi. org/10.4067/S071807052010000100004

DAY, C. Formar docentes. Cómo, cuándo y en qué condiciones aprende el profesorado. Madrid: Narcea, 2005.

FERRADA, D.; VILLENA, A.; TURRA, O. Transformar la formación. Las voces del profesorado. Concepción: Universidad Católica de la Santísima Concepción, 2015.

FLICK, Uwe. Introducción a la investigación cualitativa. Madrid: Morata, 2012.

GLASER, B. \& STRAUSS, A. The discovery of Grounded theory: Strategies for qualitative research. New York: Aldine Publishing, 1997.

GONZÁLEZ, M.; FUENTES, E. El Practicum en el aprendizaje de la profesión docente. Revista de Educación, n. 354, p. 47-70. 2011.

HIRMAS, C.; CORTÉS, I. Investigaciones sobre la formación práctica en Chile: Tensiones y desafios. Santiago de Chile: Organización de Estados Iberoamericanos para la Educación, la Ciencia y la Cultura, 2015.

LABRA, P. Construcción de conocimiento profesional docente: el caso de la formación 
en la práctica. 2011. 690 p. Tesis (Doctor en Educación) — Universidad Academia de Humanismo Cristiano, Santiago, Chile, 2011.

LATORRE, M. Prácticas pedagógicas en la encrucijada: argumentos, lógicas y razones de los actores educativos. Revista Pensamiento Educativo, v. 44-45, p. 185-210, 2009.

LORENZATTI, M. del C.; BRUMAT, M; BEINOTTI, G. Políticas de formación docente inicial para la educación rural en Argentina. Revista Iberoamericana de Evaluación Educativa, v. 7, n. 3, p. 45-55. 2014.

MARCELO, C. Aprender a enseñar para la sociedad del conocimiento. Revista Complutense de Educación, v. 12, n., p. 531-593. 2001.

MONTECINOS, C.; WALKER, H. La Colaboración entre los Centros de Práctica y las Carreras de Pedagogía. Docencia, n. 42, p. 65-73. 2010.

MONTECINOS, C.; WALKER, H.; SOLÍS, M.; NÚÑEZ, C.; CONTRERAS, I; RITTERSHAUSSEN, S. Lineamientos para el Diseño del Currículo de Formación de Carreras de Pedagogía. En MARTINIC, S. y ELAQUA, G. ¿Fin de ciclo? Cambios en la gobernanza del sistema educativo (pp. 233-256). Santiago: ORALC/UNESCO. Pontificia Universidad Católica de Chile, 2011.

SORIA, M. G.; HERNÁNDEZ, R. Aportes a la formación docente desde comunidades de aprendizaje. Revista del Cisen Tramas/Maepova, 5 (2), 131-145. 2017.

TARDIF, M. Los saberes del docente y su desarrollo profesional. Madrid: NARCEA, 2010. TURRA-DÍAZ, O.; FLORES-LUEG, C. La formación práctica desde el estudiantado de pedagogía. Ensaio: aval. pol. públ. Educ.2018. http://dx.doi.org/10.1590/s010440362018002601517

TURRA-DÍAZ, O.; FERRADA, D.; VILLENA, A. La especificidad del contexto indígena como requerimiento para la formación inicial del profesorado. Estudios Pedagógicos, v. XXXIX, n. 2, p. 329-339.2013

VERA, D.; OSSES, S.; SCHIEFELBEIN, E. Las Creencias de los profesores rurales: una tarea pendiente para la investigación educativa. Estudios Pedagógicos, v.38, n.1, p. 297-310. 2012.

VEZUB, L. Los saberes docentes en la formación inicial. La perspectiva de los formadores. Pensamiento Educativo. Revista de Investigación Educacional Latinoamericana, v.53, n. 1, p. 1-14. 2016.

ZABALZA, M. El Practicum en la formación universitaria: estado de la cuestión. Revista de Educación, n. 354, p. 21-43. 2011.

Texto recebido em 17 de outubro de 2018 .

Texto aprovado em 21 de novembro de 2018. 